\title{
The Elongation of Biceps Muscle Tendon Unit After Rerouting of the Long Head of Biceps Tendon as Superior Capsular Augmentation: A Quantitative Measurement
}

\author{
Hailong Zhang, M.D., Ph.D., and Chunyan Jiang, M.D., Ph.D.
}

\begin{abstract}
Purpose: The purpose of this study was to investigate the elongation of the biceps muscle tendon unit after rerouting of the long head of the biceps tendon without transection, with and without release of the transverse humeral ligament in a 3-dimensional model. Methods: A total of 60 sets of computed tomography images of shoulders were acquired, and a point-cloud model was generated after 3-dimensional reconstruction. Simulation of rerouting of the long head of the biceps tendon was performed by assuming that the long head of the biceps tendon travels to the center of the greater tuberosity from the supraglenoid tubercle and then back to the bicipital groove distally, with or without the release of the transverse humeral ligament. The elongation of the biceps muscle tendon unit by both manners was measured and correlated with age, height, weight, body mass index, gender, and hand dominance. Results: The mean length of the long head of the biceps tendon is $74.22 \pm 3.06 \mathrm{~mm}$, and the length is significantly longer, at $112.23 \pm 4.92 \mathrm{~mm}$ $(P=0.0002)$ and $96.47 \pm 2.58 \mathrm{~mm}(P=0.0004)$. The absolute elongation of the biceps muscle tendon unit after rerouting of the long head of the biceps tendon, without or with transverse humeral ligament release, showed significant differences, which were $38.73 \pm 3.03 \mathrm{~mm}$ and $22.28 \pm 3.25 \mathrm{~mm}$, respectively $(P=0.0008)$. No significant correlations were observed between the amount of the elongation and the subject's age, height, weight, or body mass index. No significant difference was observed in the amount of the elongation between differing gender and hand dominance. Conclusions: Rerouting of the long head of the biceps tendon resulted in significant elongation of the biceps muscle tendon unit. Release of the transverse humeral ligament partially decreased this elongation. Clinical Relevance: Rerouting of the long head of the biceps tendon may lead to overtension of the biceps. If it is adopted in clinical use, transverse humeral ligament release is recommended.
\end{abstract}

$\mathbf{M}$ assive rotator cuff tears are common pathologies that affect the aging population by causing pain and weakness of the shoulder that compromise daily function. ${ }^{1,2}$ For chronic cases with delayed presentation, severe tendon retraction and fatty infiltration in

From the Department of Sports Medicine, Beijing Jishuitan Hospital, Beijing, China.

The authors report no conflicts of interest in the authorship and publication of this article. Full ICMJE author disclosure forms are available for this article online, as supplementary material.

Received August 26, 2019; accepted June 16, 2020.

Address correspondence to Chunyan Jiang, M.D., Ph.D., Department of Sports Medicine, Beijing Jishuitan Hospital, No. 31 Xinjiekou East Street, Xicheng, Beijing, 100035, China. E-mail: chunyanj@hotmail.com

(C) 2020 THE AUTHORS. Published by Elsevier Inc. on behalf of the Arthroscopy Association of North America. This is an open access article under the CC BY-NC-ND license (http://creativecommons.org/licenses/by-nc-nd/4.0/). 2666-061X/191049

https://doi.org/10.1016/j.asmr.2020.06.007 muscle will decrease the likelihood of full repair of the tendon. ${ }^{3,4}$ To address this problem, multiple techniques have been proposed in the literature, including partial repair, tenotomy of the long head of the biceps, latissimus dorsi tendon transfer, and reverse total shoulder arthroplasty. ${ }^{5-9}$ Recently, several studies have indicated a paramount role of the superior capsule of the glenohumeral joint in stabilization of the joint, which has been shown to be disrupted in the pathology of irreparable rotator cuff tear. ${ }^{10}$ Reconstruction of the superior capsule of the glenohumeral joint, which has been suggested to restore its role in balancing the superior migration of the humeral head and maintaining the acromiohumeral distance, has been proven to be a promising technique for the management of irreparable rotator cuff tear. ${ }^{11}$

Among all the grafts used for superior capsule reconstruction, the long head of the biceps tendon (LHBT) has gained increased popularity because of the 
convenience of obtaining it, the lack of immune rejection, easier surgery, lower expenses, and favorable short to intermediate clinical outcome. ${ }^{12-14}$ However, it is still debated whether to perform tenodesis of the proximal part of biceps tendon with tenotomy of distal part or just to reroute the tendon laterally, with preservation of its continuity. It has been reported that biceps tenotomy is associated with cosmetic deformity, cramping or weakness, while maintaining the continuity of LHBT along with the muscle belly will preserve the vascularity to the tendon, which would facilitate healing. ${ }^{12,15}$ But others may have a consideration about the elongation of the biceps muscle tendon unit after rerouting, which potentially leads to an increase in the tension and anchor pullout. ${ }^{12,16}$

The purpose of this study was to investigate the elongation of the biceps muscle tendon unit after LHBT rerouting with and without release of the transverse humeral ligament in a 3-dimensional (3D) model. We hypothesized that the rerouting of the LHBT would result in significant elongation of the biceps muscle tendon unit and that release of the transverse humeral ligament could partially decrease the elongation.

\section{Methods}

\section{3-D Computed Tomography Model Reconstruction and Identification of the Landmarks for LHBT Rerouting}

The study was approved by the institutional review board, and informed consent was obtained from each patient. Computed tomography (CT) scan images (Aquilion 64, Toshiba America Medical Systems, Tustin, CA, USA) were obtained from a total of 60 patients ( 35 female and 25 male, 37 right shoulder and 23 left shoulder) between January 2015 and October 2019. The mean age of patients enrolled was 66 years (range 54-73 years), and all the patients were right-handed. Inclusion criteria were patients with massive rotator cuff tear without bony changes evidenced by CT. Exclusion criteria included patients with fracture or deformity in the scapula or proximal humerus.

In our study, the standard $120 \mathrm{kVp}$ and $200 \mathrm{~mA}$ bonereconstruction sequence was employed, with the parameters set at a slice thickness of $0.8 \mathrm{~mm}$ and a resolution of $512 \times 512$ pixels. The upper arm was immobilized in neutral position during scanning. The 3-D model of the scapula and proximal humerus was reconstructed from the CT images using commercial 3-D reconstruction software (Mimics, Materialise, Leuven, Belgium) (Fig 1). To measure the length and elongation of the biceps muscle tendon unit, 4 anatomic landmarks were identified on the model, including the supraglenoid tubercle, the center of greater tuberosity, the superior margin of intertubercular groove, and the inferior margin of the intertubercular groove. The supraglenoid tubercle was marked, with the most prominent point at the supraglenoid tubercle, while the center of greater tuberosity was identified as the midpoint of the lateral margin of the footprint on the greater tuberosity. The superior margin of the intertubercular groove was identified as a point at the level of the lesser tuberosity within the groove, while the inferior margin of intertubercular groove was marked as a point within the groove and $52 \mathrm{~mm}$ below the top of the humeral head, which is the superior insertion of pectoralis major ${ }^{17}$ (Fig 1). For each of the anatomic landmarks, coordinates in the 3-D frame $(\mathrm{x}, \mathrm{y}, \mathrm{z})$ were recorded for subsequent analysis. The model was independently viewed and marked by 2 sports medicine surgeons (CJ, HZ) on 2 separate occasions at a mean of 3 weeks apart.

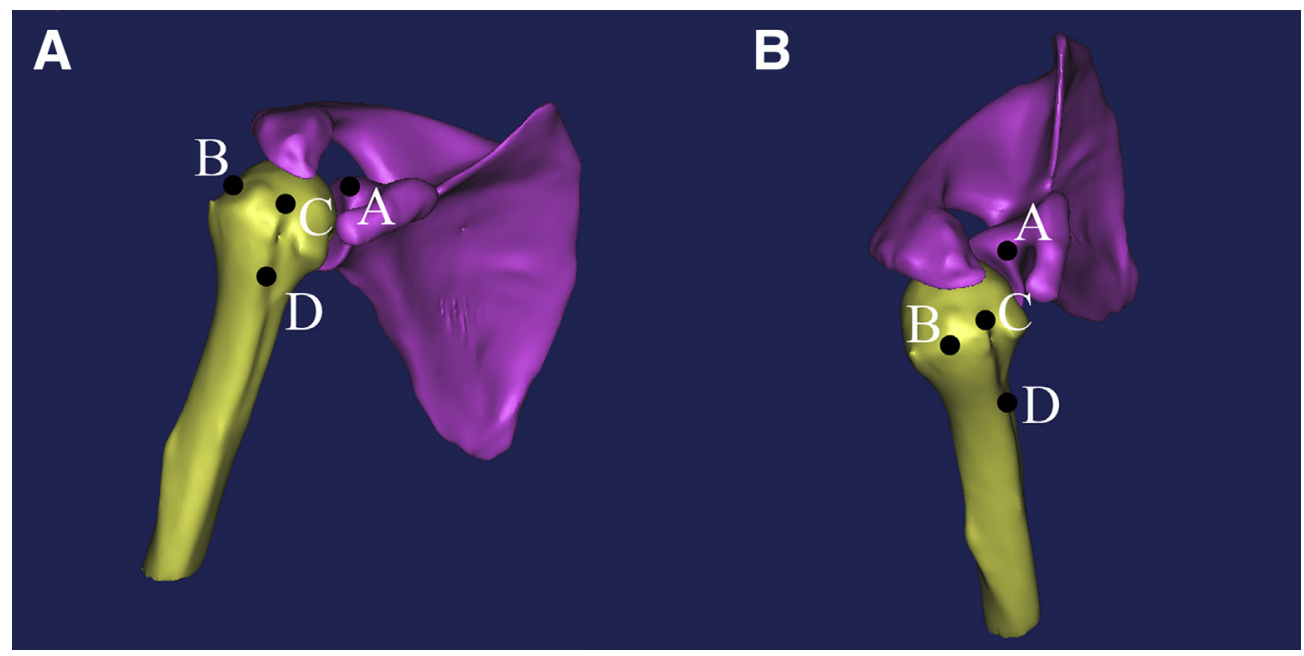

Fig 1. Illustration of the anatomic landmarks for LHBT rerouting on right shoulder 3-D model of the glenoid and the proximal humerus and illustration of the anatomic landmarks for LHBT rerouting. (A) 3-D contour of the glenoid and the proximal humus was illustrated. (B) Bird's-eye view is employed to show the footprint of supraspinatus and infraspinatus on greater tuberosity. A, supraglenoid tubercle; $\mathrm{B}$, the center of greater tuberosity; C, the entrance into intertubercular groove; $\mathrm{D}$, the expected most superior insertion of pectoralis major. (LHBT, long head of biceps tendon.) 

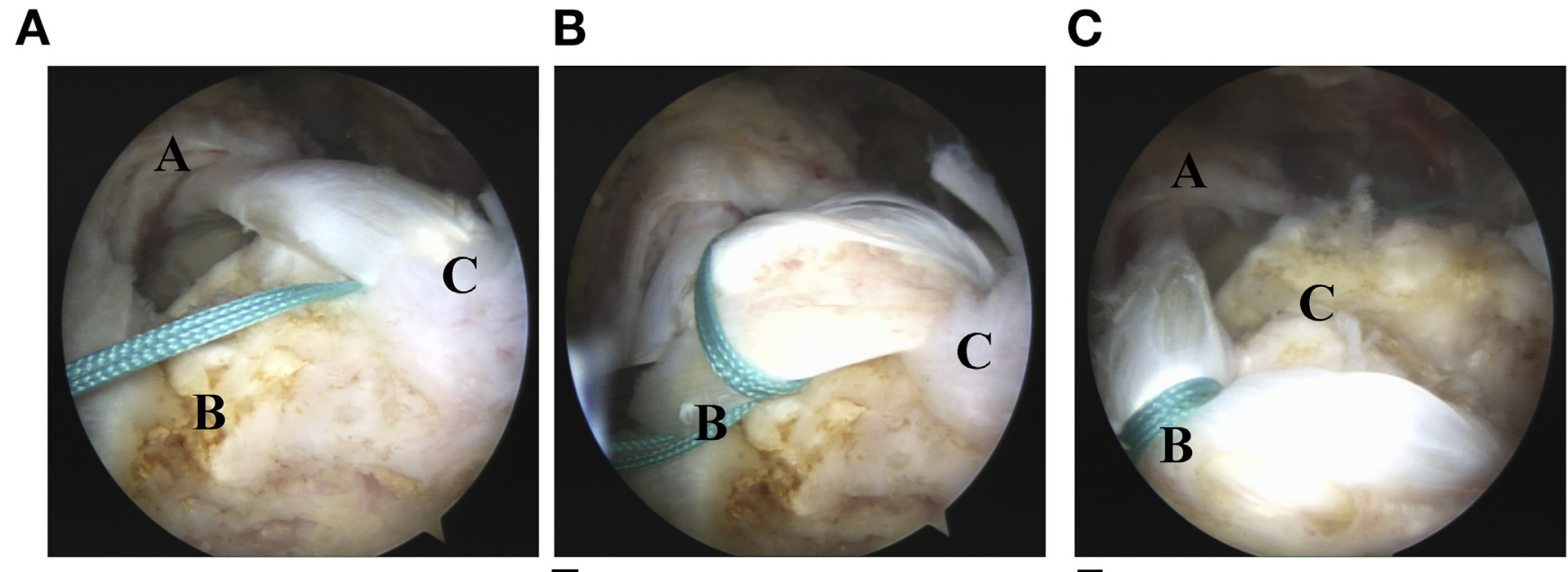

D


$\mathbf{F}$

Fig 2. Intraoperative view and simulation of LHBT rerouting of right shoulder. (A, D) Original path of LHBT from supraglenoid tubercle via entrance into intertubercular groove to the level of the insertion of pectoralis major. This was the sum of length of arc AC and CD. (B, E) Rerouting of LHBT to greater tuberosity without release of transverse humeral ligament. The path of LHBT went from supraglenoid tubercle to the midpoint of the lateral margin of the footprint of greater tuberosity and then went back to the entrance into the intertubercular groove until the level of the insertion of the pectoralis major. This was the sum of length of arc $\mathrm{AB}, \mathrm{BC}$ and $\mathrm{CD}$. (C, F) Rerouting of LHBT with transverse humeral ligament. The path of LHBT was from supraglenoid tubercle to the midpoint of the lateral margin of the footprint of the greater tuberosity and was then directly aimed to the level of the insertion of the pectoralis major. This was the sum of length of arc AB and BD. A, supraglenoid tubercle; B, the center of greater tuberosity; $\mathrm{C}$, the entrance into intertubercular groove; D, the expected most superior insertion of pectoralis major. (LHBT, long head of biceps tendon.)

\section{3-D Point-cloud Model Generation and Simulation of LHBT Rerouting}

The reconstructed 3-D model of the glenoid and proximal humerus associated with the identified anatomic landmarks was exported in the format of a point-cloud pattern and then imported into MatLab software (Mathworks, Natick, MA, USA) to generate the point-cloud model for the following analysis. The radius of the humeral head was determined by fitting the humeral head using the best-fit sphere.

To simulate the rerouting of the LHBT, 3 conditions were tested. First, the original path of the LHBT was marked; it led from the supraglenoid tubercle to the entrance of the bicipital groove and through the intertubercular groove to the level of the expected, most superior insertion of the pectoralis major, where it was regarded as the constant landmark for subsequent comparison (Fig 2A, D). Second, to use the autologous
LHBT for superior capsule reconstruction, it was rerouted to the center of the greater tuberosity to restore a full coverage by the supraspinatus. Then it went back to the entrance into the intertubercular groove until the level of the expected most superior insertion of the pectoralis major (Fig 2B, E). Third, if transverse humeral ligament release was further performed, the path of the LHBT would travel directly to the level of the expected most superior insertion of the pectoralis major after leaving the greater tuberosity without going through the intertubercular groove (Fig 2C, F). The length of the LHBT was defined as the total length from the supraglenoid tubercle to the level of the expected most superior insertion of the pectoralis major via various courses. The length of the LHBT under each setting was measured, and the elongation of the biceps muscle tendon unit after the rerouting was compared. 


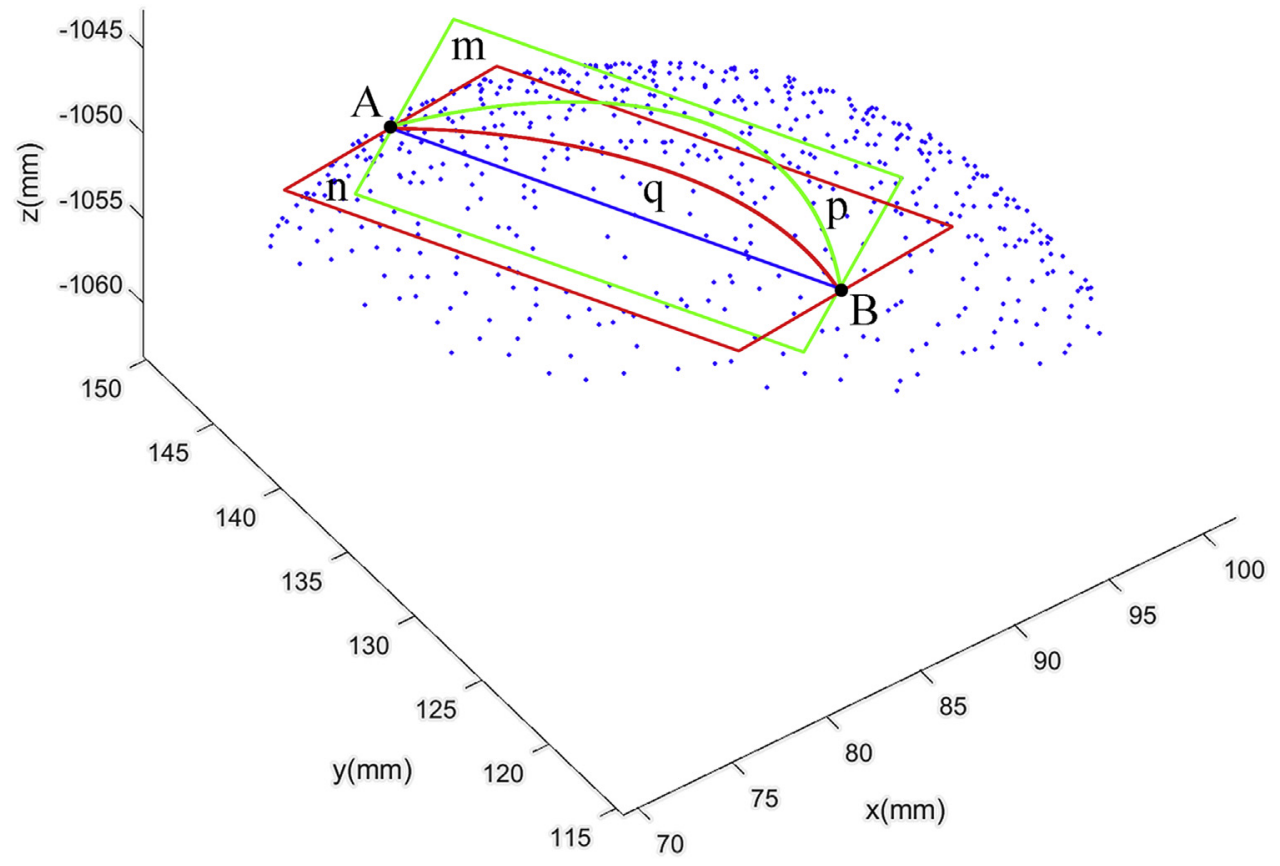

Fig 3. Measurement of the 3-D distance between 2 points on an irregular surface. Point $\mathrm{A}$ and $\mathrm{B}$ are 2 points on an irregular surface (blue dots) with a line passing $\mathrm{A}$ and $\mathrm{B}$ (blue line). To illustrate the algorithm for calculation of the 3-D distance between point $A$ and $B$, a set of planes passing line $A B$ with an angular increment of $5^{\circ}$ is created. Here only 2 planes, $m$ and $n$, were drawn for demonstration. Planes $m$ and $n$ intersect the irregular surface separately and produce a curve, $\mathrm{p}$ and $\mathrm{q}$. The length of each curve is calculated, and the smallest value is adopted as the 3-D distance between points A and $\mathrm{B}$ on the surface.

\section{Measurement of the 3-D Distance Between Two Points on an Irregular Surface}

A key step in the calculation of the length of the LHBT in differing settings is to measure accurately the distance between 2 points on an irregular surface in a 3-D frame. In this study, a least-distance algorithm was developed with the principle of calculus of variation. Basically, set points A and B (blue dots in Fig 3) are 2 points on an irregular surface, with a line passing $A$ and $B$. A set of planes passing line $A B$ with an angular increment of $5^{\circ}$ is created, and each plane produces a curve by intersection with the surface. The length of each curve is calculated, and the smallest value is adopted as the 3 -D distance between 2 points on the surface (Fig 3).

\section{Statistical Analysis}

All the data are expressed as the mean and standard deviation. Shapiro-Wilk tests were performed to check normality of all variables. The values of elongation of the biceps muscle tendon unit in differing settings were compared and examined using the paired-samples Student $t$ test. Spearman rank correlation tests were used to investigate the relationship between the elongation of the biceps muscle tendon unit without or with transverse humeral ligament release and the demographic factors, including age, height, weight, and body mass index (BMI). The Student $t$ test was used to evaluate the difference in the elongation, without or with transverse humeral ligament release regarding gender and hand dominance. The intraobserver reliability and interobserver reliability of the identified coordinates of the anatomic landmarks were evaluated by intraclass correlation coefficients (ICCs), which were classified as excellent (ICC $>0.75$ ), good (ICC 0.60-0.74), fair (ICC 0.40-0.59), or poor (ICC $<0.40) .{ }^{18}$ Significant difference was considered when the $P$ value was less than 0.05 . A sample size of 60 patients was adequate to achieve $80 \%$ power regarding the comparison.

\section{Results}

The mean length of the LHBT from the supraglenoid tubercle to the expected superior-most insertion of pectoralis major is $74.22 \pm 3.06 \mathrm{~mm}$, while the length is significantly increased to $112.23 \pm 4.92 \mathrm{~mm}$ $(P=0.0002)$ and $96.47 \pm 2.58 \mathrm{~mm}(P=0.0004)$ after rerouting to the greater tuberosity without or with transverse humeral ligament release (Fig 4). The absolute elongation of the biceps muscle tendon unit after rerouting of the LHBT, without or with transverse humeral ligament release, showed significant difference: $38.73 \pm 3.03 \mathrm{~mm}$ and $22.28 \pm 3.25 \mathrm{~mm}$, respectively $(P=0.0008)$ (Fig 4).

Significant correlation was obtained between the amount of the elongation and the radius of the humeral head, whereas no significance was observed between the amount of the elongation and the subject's age, height, weight, or BMI (for simulation without release of transverse humeral ligament, correlation with radius of humeral head: rho $=0.54, P=0.0007$; age: rho $=0.08, P=0.15$; height: rho $=0.17, P=0.35$; weight: rho $=0.32, P=0.47$; $\mathrm{BMI}$, rho $=0.27, P=0.24$; 


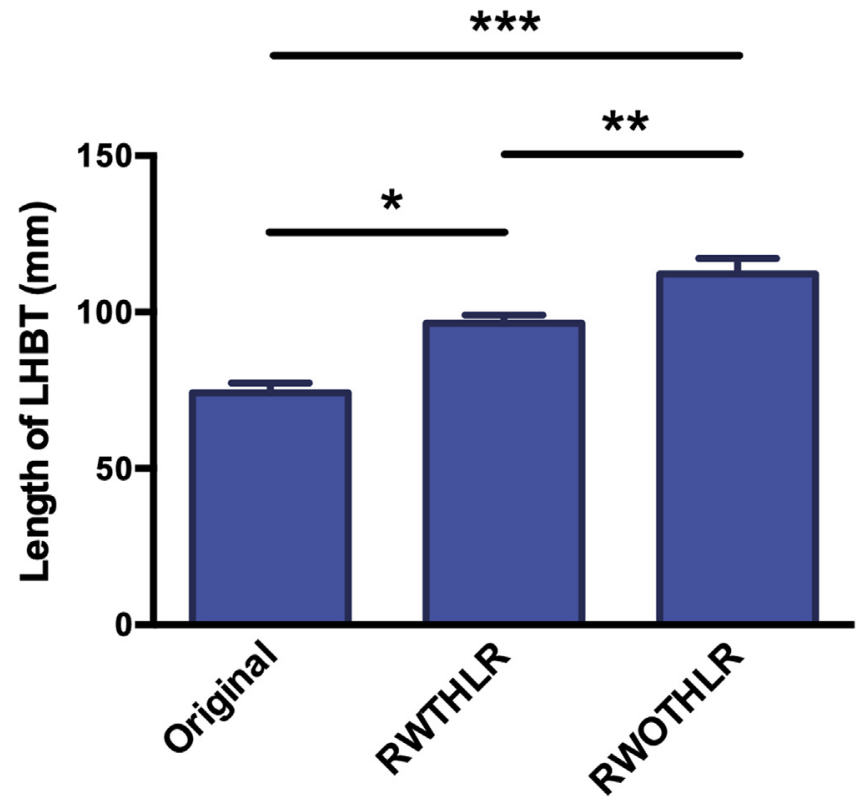

Fig 4. The length of the LHBT before and after rerouting. The figure shows the length of the LHBT before and after the rerouting. (LHBT, long head of biceps tendon; RWOTHLR, rerouting without transverse humeral ligament release; RWTHLR, rerouting with transverse humeral ligament release.) *, 0.0007; **, $0.0004 ; * * *, 0.0002$

for simulation with release of the transverse humeral ligament, correlation with the radius of the humeral head: rho $=0.22, P=0.001$; age: rho $=0.13, P=0.25$; height: rho $=0.37, P=0.35$; weight: rho $=0.48$, $P=0.17$; BMI, rho $=0.44, P=0.34$ ). No significant difference was observed in the amount of the elongation between different genders and hand dominance (for simulation without release of transverse humeral ligament, gender: $P=0.27$; hand dominance: $P=0.32$; for simulation with release of transverse humeral ligament, gender: $P=0.45$; hand dominance: $P=0.13$ ).

Physicians' agreement in identification of the anatomic landmarks between the 2 views of the same patient was excellent, with an ICC ranging from 0.78 to 0.91 $(P=0.0007)$. The agreement in identification of the anatomic landmarks among various physicians was excellent, with an ICC ranging from 0.71 to 0.84 $(P=0.0008)$.

\section{Discussion}

Rerouting of the LHBT would result in significant elongation of the biceps muscle tendon unit, and release of the transverse humeral ligament could partially decrease the elongation.

Surgical treatment of massive rotator cuff tear remains a challenge for shoulder surgeons. Various techniques have been tried so as to improve the clinical outcome. Recently, superior capsular reconstruction was reported to be an effective treatment for massive rotator cuff tear by recentering the humeral head to prevent superior migration. ${ }^{1,10}$ Mihata and colleagues' result suggested an improvement in pain, range of motion and strength in their short-term follow-up study. ${ }^{10}$ Several graft resources have been suggested for the reconstruction of the superior capsule. In Mihata et al.'s original research, they used fascia lata autograft. ${ }^{10}$ Although fascia lata harvest is a simple procedure, it may result in addition donor-site morbidity, such as pain on walking, scarring, scar sensitivity, hematoma, muscle herniation, and wound infection. ${ }^{19,20}$ To avoid the possible donor-site morbidity, some authors have proposed human acellular dermal allograft as an alternative. ${ }^{14,21}$ Despite the growing popularity of use of this new material, the immune reaction and the price are major concerns in their application. $^{22}$ To avoid the above complication, the LHBT was proposed as an excellent source because of the advantage of natural available tissue, reduced cost, a less technically demanding procedure, and no donor-site comorbidity. ${ }^{1,22}$

Even though the application of the LHBT as a source of superior capsular reconstruction has gained popularity in recent years, the exact procedure has not been agreed on.

First, where should the tendon fixation site be? In Lin's study, they inserted the anchor for fixation close to the entrance of intertubercular groove, ${ }^{13}$ whereas Chillemi suggested that the point $5-10 \mathrm{~mm}$ lateral to the greater tuberosity was the best place. ${ }^{23}$ A study performed by Kim used the point between footprint and cartilage as the fixation site. ${ }^{17}$ In our study, we adopted the method developed by Hermanowicz, who believed that the center of the greater tuberosity resembled the anatomic insertion of the supraspinatus, which is the optimal location for anchor insertion. ${ }^{16}$

Second, should the continuity of the LHBT be preserved? To date, little of the literature has focused on the comparison between LHBT tenodesis and distal tenotomy with LHBT rerouting. The reasons for preserving the continuity of the biceps originated from the benefits of the tendon biology because maintaining the integrity of the biceps would provide better blood supply and benefit the healing process. Moreover, the intact LHBT may serve as a vascularized graft for the retracted supraspinatus and infraspinatus, which bridge the torn end to the greater tuberosity. ${ }^{16}$

Third, should the transverse humeral ligament be released? Prior studies have suggested that the transverse humeral ligament is a distinct structure covering the intertubercular groove, which is composed of the fibers of the subscapularis tendon, with contributions from the supraspinatus tendon and the coracohumeral ligament. ${ }^{24-27}$ There may be concern about the risk of subscapularis tendon damage after transverse humeral ligament release. However, this procedure has been widely used in LHBT tenodesis with excellent clinical outcomes. ${ }^{27,28}$ 
Regarding the consequence of elongation of the biceps muscle tendon unit, it has been reported that elongation of the biceps muscle tendon unit after distal biceps repair leads to various outcomes in the Disabilities of the Arm, Shoulder and Hand (DASH) score. ${ }^{29}$ There have also been concerns about the increased tension caused by rerouting of the LHBT because overtension of the biceps muscle tendon unit may lead to failure through anchor pullout or tendon rupture. ${ }^{30}$ This is proved by our study. We found that rerouting of the LHBT would result in elongation of the biceps muscle tendon unit to an average of $3.9 \mathrm{~cm}$. This overtension of the biceps muscle tendon unit may cause clinical symptoms and put the fixation into high risk of failure.

Most of the procedure of LHBT tenodesis or rerouting requires release of the transverse humeral ligament to enable better visualization of the site for anchor insertion. ${ }^{12,16}$ However, few investigations have been conducted to evaluate the role of releasing the transverse humeral ligament in the elongation of the biceps muscle tendon unit after rerouting of the LHBT in treatment of massive rotator cuff tears. ${ }^{16}$ Our results suggested that release of the transverse humeral ligament would decrease the elongation of biceps muscle tendon unit, which may consequently reduce the tension of in the biceps after the procedure. The strength of our study is that, with the help of 3-D modeling, we were measuring the true 3 -D distance between 2 points on an irregular surface. This made our result more accurate and reliable compared with simple 2-D measurement.

\section{Limitations}

There are several limitations to our study. First, this study was performed on a 3-D reconstruction model from CT images instead of measurement in situ. However, because the course of the LHBT is just on the surface of the bone architecture, the thickness of soft tissue such as the periosteum was minimal. Second, our measurement was conducted when the shoulder was placed in $0^{\circ}$ of abduction and neutral position, which is different from the posture in the beach-chair position or the lateral decubitus position intraoperatively. Third, internal or external rotation of the upper arm may result in changes in the position of the anatomic markers for the evaluation which, in turn, would have an effect on the final measurement. It was suggested that internal rotation would decrease the total length of the biceps muscle tendon unit, whereas external rotation would increase the overall length of the biceps muscle tendon unit. In this study, the upper arm was placed in a neutral position during the scanning so as to diminish the effect of rotation. Another limitation is that the anatomic landmarks for calculation were identified manually, which may bring in variation.
However, the reliability between the 2 independent observers was excellent. It was admitted that this study only quantitatively evaluated the elongation of the biceps muscle tendon unit in distance after rerouting, whereas soft-tissue deformation, collagen elasticity and tension generated after the elongation were not measured in this geometric model.

\section{Conclusions}

Rerouting the LHBT resulted in significant elongation of the biceps muscle tendon unit. Release of the transverse humeral ligament partially decreased this elongation.

\section{Acknowledgment}

The authors thank Dr. Na Clara Pan from Institute of biophysics, Chinese Academy of Science, for helpful discussions and comments on the manuscript.

\section{References}

1. Han F, Kong CH, Hasan MY, et al. Superior capsular reconstruction for irreparable supraspinatus tendon tears using the long head of biceps: A biomechanical study on cadavers. Orthop Traumatol Surg Res 2019;105:257-263.

2. Kim SJ, Lee IS, Kim SH, et al. Arthroscopic partial repair of irreparable large to massive rotator cuff tears. Arthroscopy 2012;28:761-768.

3. Bedi A, Dines J, Warren RF, Dines DM. Massive tears of the rotator cuff. J Bone Joint Surg Am 2010;92:1894-1908.

4. Oh JH, Kim SH, Kang JY, et al. Effect of age on functional and structural outcome after rotator cuff repair. Am J Sports Med 2010;38:672-678.

5. Boileau P, Baqué F, Valerio L, et al. Isolated arthroscopic biceps tenotomy or tenodesis improves symptoms in patients with massive irreparable rotator cuff tears. J Bone Joint Surg Am 2007;89:747-757.

6. Guery J, Favard L, Sirveaux F, et al. Reverse total shoulder arthroplasty: Survivorship analysis of eighty replacements followed for five to ten years. J Bone Joint Surg Am 2006;88:1742-1747.

7. Kanatlı U, Özer M, Ataoğlu MB, et al. Arthroscopicassisted latissimus dorsi tendon transfer for massive, irreparable rotator cuff tears: Technique and short-term follow-up of patients with pseudoparalysis. Arthroscopy 2017;33:929-937.

8. Kim SJ, Kim SH, Lee SK, et al. Arthroscopic repair of massive contracted rotator cuff tears: Aggressive release with anterior and posterior interval slides do not improve cuff healing and integrity. J Bone Joint Surg Am 2013;95: 1482-1488.

9. Novi M, Kumar A, Paladini P, et al. Irreparable rotator cuff tears: Challenges and solutions. Orthop Res Rev 2018;10: 93-103.

10. Mihata T, McGarry MH, Pirolo JM, et al. Superior capsule reconstruction to restore superior stability in irreparable rotator cuff tears: A biomechanical cadaveric study. Am J Sports Med 2012;40:2248-2255. 
11. Mihata T, Lee TQ, Watanabe C, et al. Clinical results of arthroscopic superior capsule reconstruction for irreparable rotator cuff tears. Arthroscopy 2013;29:459-470.

12. Kim YS, Lee HJ, Park I, et al. Arthroscopic in situ superior capsular reconstruction using the long head of the biceps tendon. Arthrosc Tech 2018;7:e97-e103.

13. Lin J, Qi W, Liu Z, et al. An arthroscopic technique for full-thickness rotator cuff repair by transposition of the long head of biceps. Orthop Traumatol Surg Res 2019;105: 265-269.

14. Pennington WT, Bartz BA, Pauli JM, et al. Arthroscopic superior capsular reconstruction with acellular dermal allograft for the treatment of massive irreparable rotator cuff tears: Short-term clinical outcomes and the radiographic parameter of superior capsular distance. Arthroscopy 2018;34:1764-1773.

15. Meeks BD, Meeks NM, Froehle AW, et al. Patient satisfaction after biceps tenotomy. Orthop J Sports Med 2017;5: 2325967117707737.

16. Hermanowicz K, Góralczyk A, Malinowski K, et al. Long head biceps tendon-natural patch for massive irreparable rotator cuff tears. Arthrosc Tech 2018;7:e473-e478.

17. Kim BS, Kim DH, Song KS, et al. Is the pectoralis major tendon a reliable reference for restoration of humeral length with fracture hemiarthroplasty? J Shoulder Elbow Surg 2018;27:e45-e49.

18. Cicchetti DV, Sparrow SA. Developing criteria for establishing interrater reliability of specific items: Applications to assessment of adaptive behavior. Am J Ment Defic 1981;86:127-137.

19. Dubiel WT, Wigren A. Functional status of the lower extremity after resection of fascia lata: A clinical and physiological follow-up study in patients with fascia lata heart valve replacement. Acta Orthop Scand 1974;45:599-613.

20. Wheatcroft SM, Vardy SJ, Tyers AG. Complications of fascia lata harvesting for ptosis surgery. $\mathrm{Br} J$ Ophthalmol 1997;81:581-583.
21. Hirahara AM, Adams CR. Arthroscopic superior capsular reconstruction for treatment of massive irreparable rotator cuff tears. Arthrosc Tech 2015;4:e637-e641.

22. Boutsiadis A, Chen S, Jiang C, et al. Long head of the biceps as a suitable available local tissue autograft for superior capsular reconstruction: "The Chinese Way." Arthrosc Tech 2017;6:e1559-e1566.

23. Chillemi C, Mantovani M, Gigante A. Superior capsular reconstruction of the shoulder: The ABC (Arthroscopic Biceps Chillemi) technique. Eur J Orthop Surg Traumatol 2018;28:1215-1223.

24. Gleason PD, Beall DP, Sanders TG, et al. The transverse humeral ligament: A separate anatomical structure or a continuation of the osseous attachment of the rotator cuff? Am J Sports Med 2006;34:72-77.

25. MacDonald K, Bridger J, Cash C, Parkin I. Transverse humeral ligament: Does it exist? Clin Anat 2007;20:663-667.

26. Snow BJ, Narvy SJ, Omid R, et al. Anatomy and histology of the transverse humeral ligament. Orthopedics 2013;36: el295-el298.

27. Forsythe B, Agarwalla A, Puzzitiello RN, et al. Rates and risk factors for revision open and arthroscopic proximal biceps tenodesis. Orthop J Sports Med 2019;7: 2325967118825473.

28. Werner BC, Evans CL, Holzgrefe RE, et al. Arthroscopic suprapectoral and open subpectoral biceps tenodesis: A comparison of minimum 2-year clinical outcomes. Am J Sports Med 2014:42:2583-2590.

29. Marshall NE, Keller RA, Okoroha K, et al. Radiostereometric evaluation of tendon elongation after distal biceps repair. Orthop J Sports Med 2016;4: 2325967116672620.

30. Werner BC, Lyons ML, Evans CL, et al. Arthroscopic suprapectoral and open subpectoral biceps tenodesis: A comparison of restoration of length-tension and mechanical strength between techniques. Arthroscopy 2015;31:620-627. 